\title{
Fabrication of Hierarchical Porous Carbon Frameworks from Metal-Ion-Assisted Step-Activation of Biomass for Supercapacitors with Ultrahigh Capacitance
}

Chengshuai Chang, ${ }^{\dagger},+$ He Wang, ${ }^{\dagger}$ Y Yunqiang Zhang, ${ }^{\dagger}$ Shulan Wang, ${ }^{*},{ }^{\dagger}$ Xuan Liu* ${ }^{*},{ }^{\dagger}$ and $\mathrm{Li} \mathrm{Li}^{*}, *$

$\dagger$ Department of Chemistry, School of Science, Northeastern University, Wenhua Road, Heping District, Shenyang 110819, P. R. China

$\$$ School of Metallurgy, Northeastern University, Wenhua Road, Heping District, Shenyang 110819, P. R. China

Address all correspondence to the author.

*Email: slwang@mail.neu.edu.cn (Shulan Wang); xuanliucmu@gmail.com (Xuan Liu); lilicmu@alumni.cmu.edu, lilicmu@gmail.com (Li Li)

Number of Pages: 13

Number of Figures: 5

Number of Tables: 5 


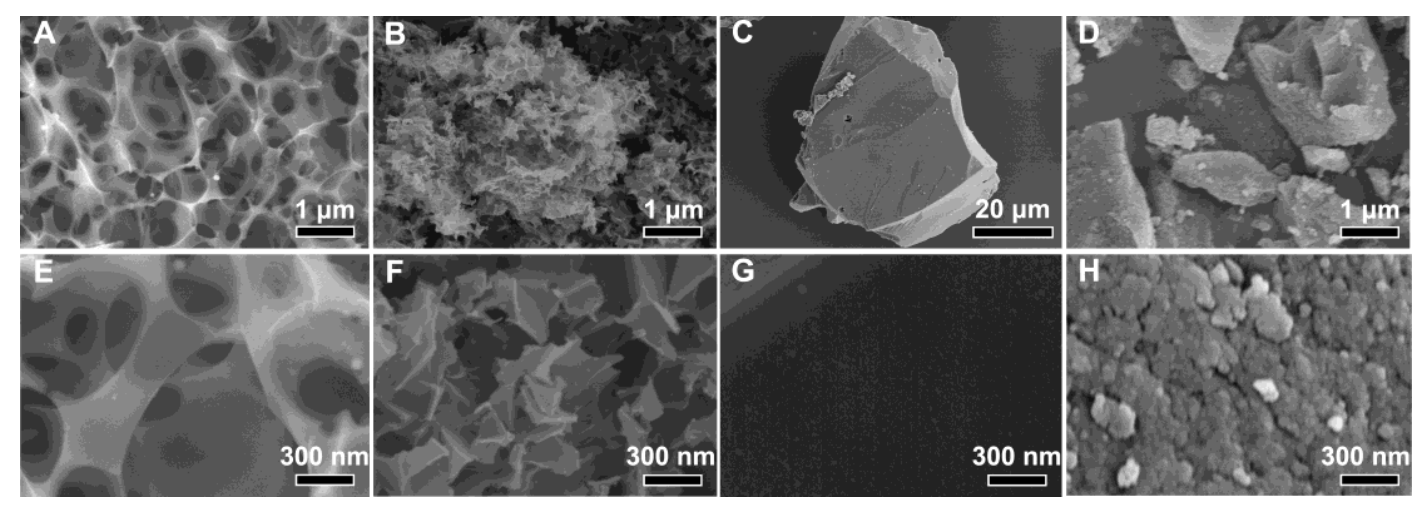

Fig. S1 SEM images of (A, E) KCPF; (B, F) NCPF; (C, G) ZCPF and (D, H) PCPF. 

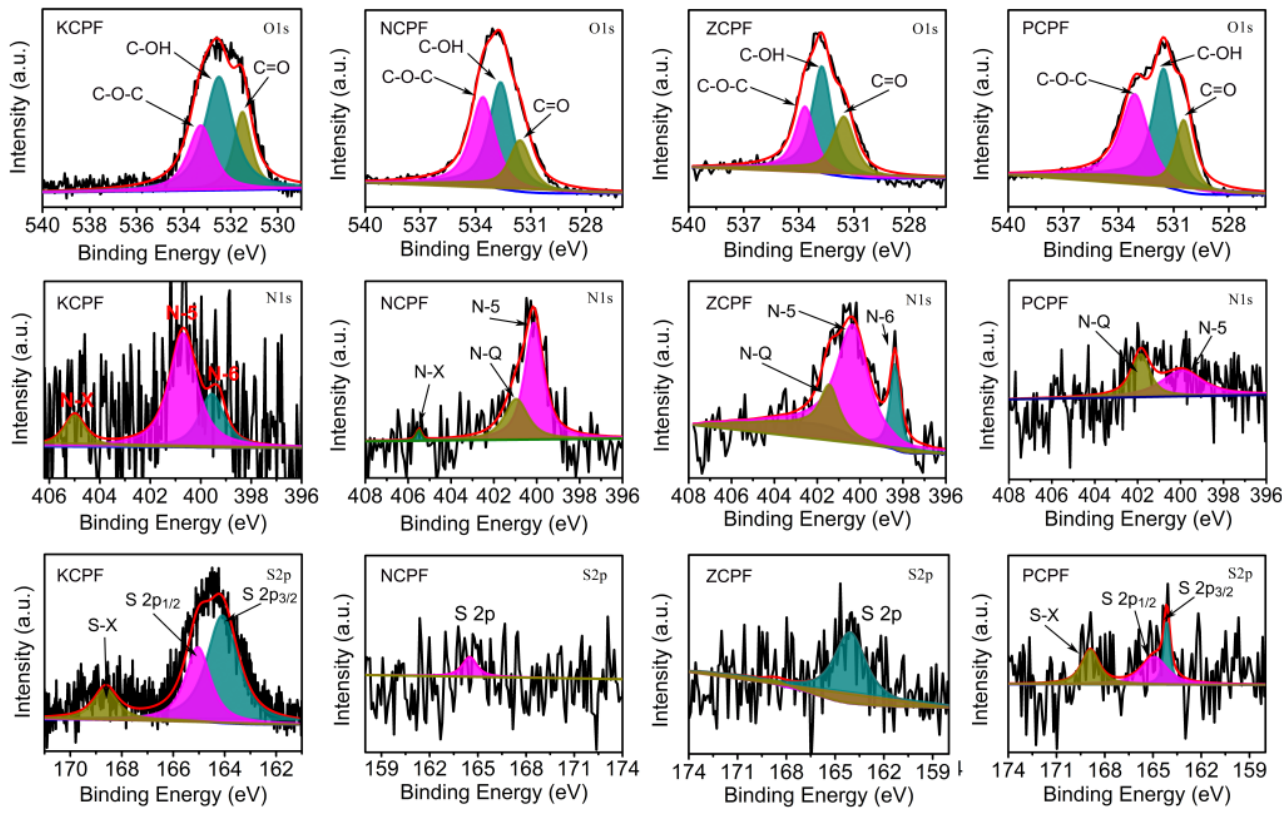

Fig. S2 High-resolution XPS scans for N 1s, O 1s and S 2p of KCPF, NCPF, ZCPF and PCPF. 

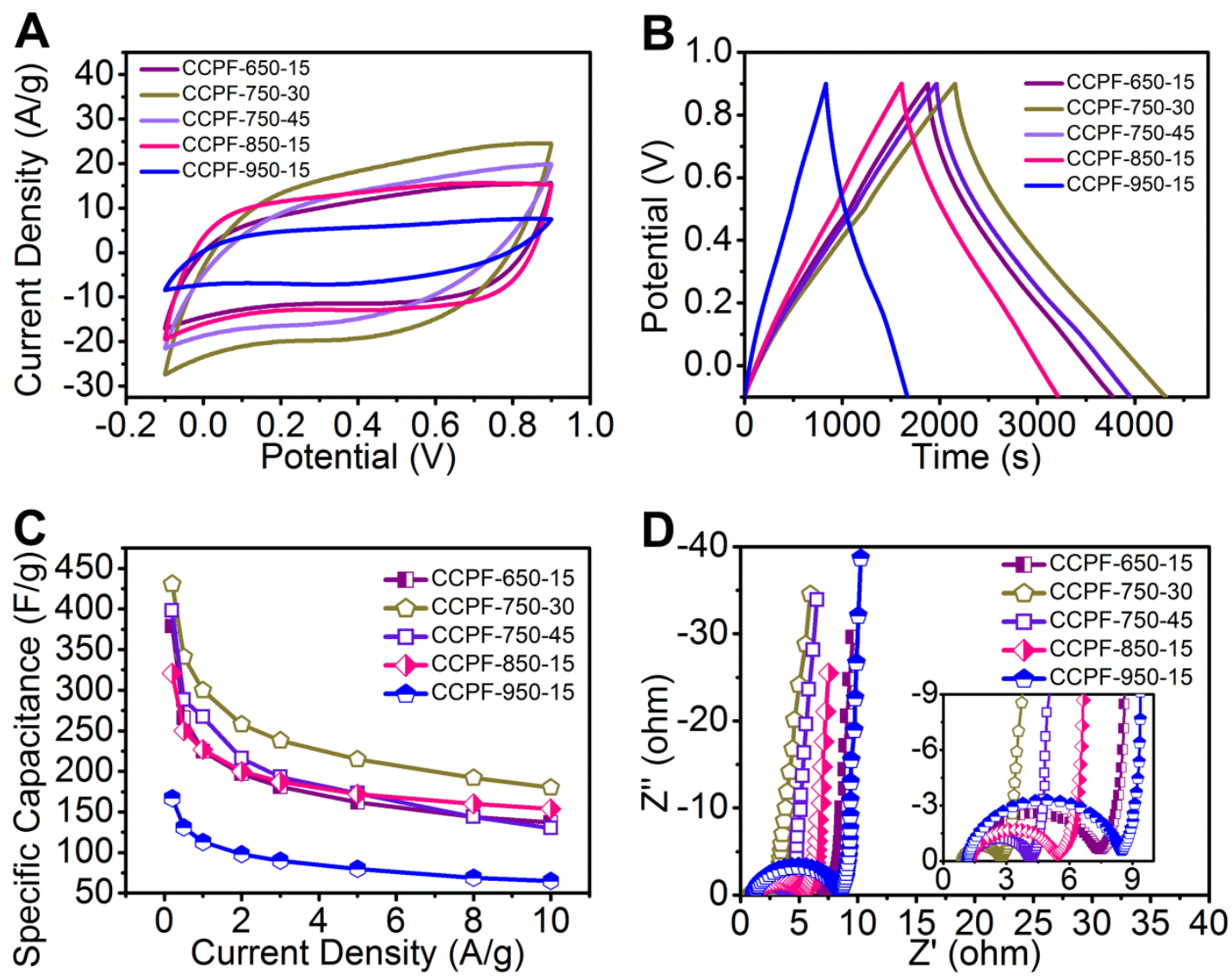

Fig. S3 Electrochemical performance of $\mathrm{CO}_{2}$ activated samples in $2 \mathrm{M} \mathrm{H}_{2} \mathrm{SO}_{4}$ solution in a three-electrode system: (A) CVs at a scan rate of $100 \mathrm{mV} \mathrm{s}^{-1}$; (B) GCD curves at the current density of $0.2 \mathrm{~A} \mathrm{~g}^{-1}$; (C) Specific capacitance at different current densities and (D) Nyquist plots. 


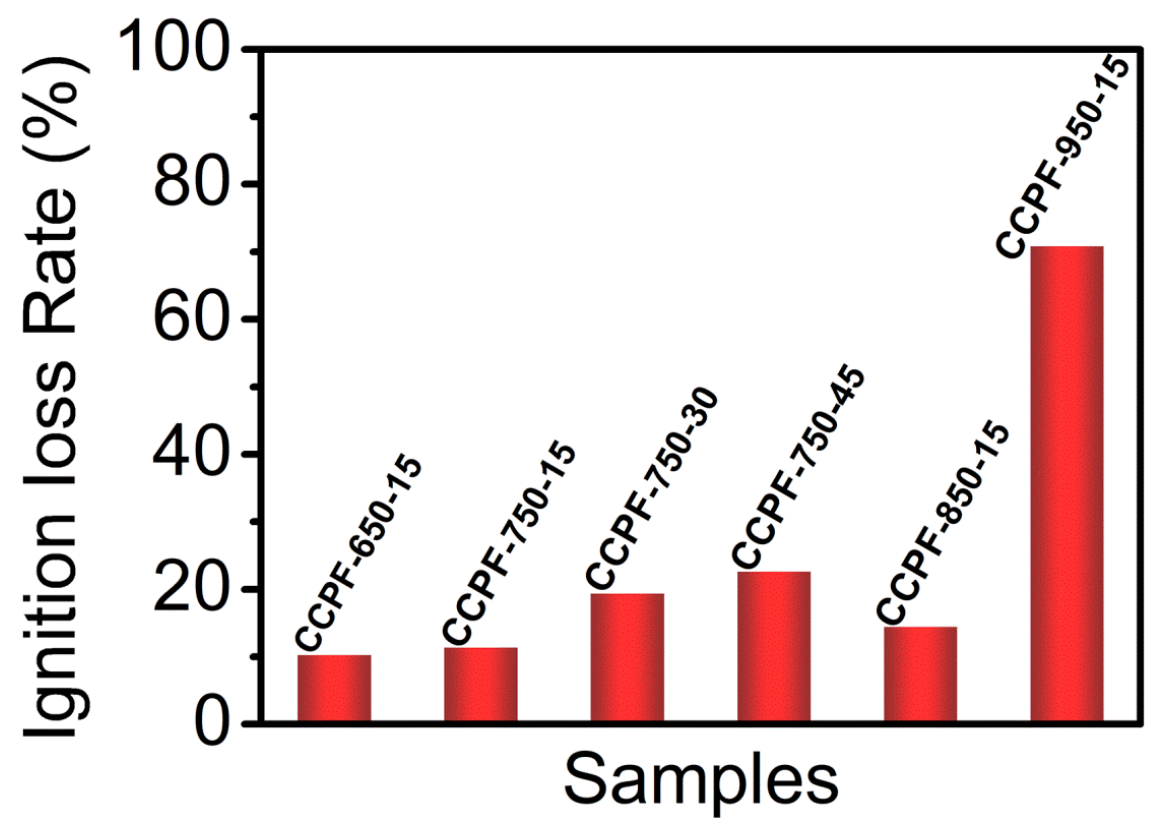

Fig. S4 Ignition loss rate of $\mathrm{CO}_{2}$ activated samples. 

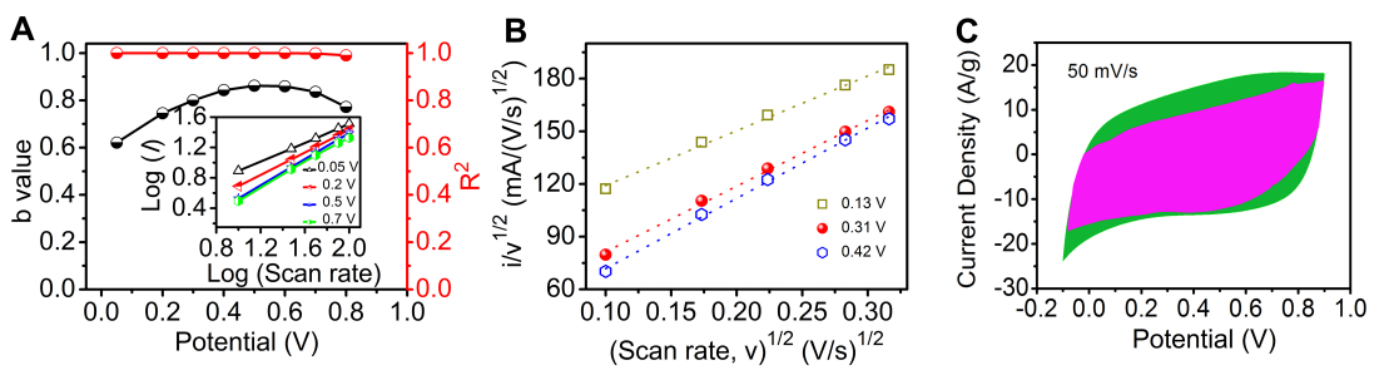

Fig. S5 CCPF-750-15 in $2 \mathrm{M} \mathrm{H}_{2} \mathrm{SO}_{4}$ electrolyte (A) b value against voltage. The inset is the plots of current response against scan rate at different voltages; (B) Plots of $i / v^{1 / 2}$ vs. $v^{1 / 2}$ for calculating $k_{1}$ and $k_{2}$; (C) capacitive contribution of surface controlled (magenta) and diffusion-controlled (green) at $50 \mathrm{mV} / \mathrm{s}$. 
Table S1 Element C, O, N and S content (atom\%) obtained from XPS.

\begin{tabular}{lcccc}
\hline \multirow{2}{*}{ The as-prepared } & \multicolumn{3}{c}{ Element content (atom\%) } & \\
\cline { 2 - 4 } \multicolumn{1}{c}{ samples } & $\mathbf{C}$ & $\mathbf{O}$ & $\mathbf{N}$ & $\mathbf{S}$ \\
\hline KCPF & 84.93 & 11.82 & 1.50 & 1.75 \\
NCPF & 87.91 & 10.84 & 0.97 & 0.27 \\
ZCPF & 86.99 & 9.98 & 2.52 & 0.51 \\
PCPF & 87.33 & 10.53 & 1.40 & 0.74 \\
CCPF-750-15 & 86.67 & 10.24 & 1.37 & 1.72 \\
\hline
\end{tabular}


Table S2 The detailed information of the reported biochar sample.

\begin{tabular}{|c|c|c|c|c|}
\hline Precursor & $\begin{array}{l}\text { Treatment } \\
\text { conditions }\end{array}$ & $\begin{array}{c}\text { SSA } \\
\left(\mathbf{m}^{2} / \mathbf{g}\right)\end{array}$ & Electrolyte & $\begin{array}{c}\text { Capacitance } \\
(\mathrm{F} / \mathrm{g})\end{array}$ \\
\hline Tobacco rods & $\begin{array}{l}\text { Hydrothermal carbonization/KOH } \\
\text { activation }\end{array}$ & 2115 & $6 \mathrm{M} \mathrm{KOH}$ & $287(0.5 \mathrm{~A} / \mathrm{g})$ \\
\hline $\begin{array}{c}\text { Glucose@ rice } \\
\text { straw }\end{array}$ & In situ decoration/KOH activation & 1122 & $6 \mathrm{M} \mathrm{KOH}$ & $337(1.0 \mathrm{~A} / \mathrm{g})$ \\
\hline Rice husk & $\begin{array}{c}\text { Pre-carbonization } / \mathrm{MgO}, \mathrm{CTP}, \mathrm{KOH} \\
\text { activation }\end{array}$ & 3120 & $6 \mathrm{M} \mathrm{KOH}$ & $315(0.1 \mathrm{~A} / \mathrm{g})$ \\
\hline Rice straw & Carbonization/KOH activation & 1007 & $1 \mathrm{M} \mathrm{H}_{2} \mathrm{SO}_{4}$ & $332(0.5 \mathrm{~A} / \mathrm{g})$ \\
\hline Cotton stalk & $\mathrm{KOH}$-chemical activation & 1964 & $1 \mathrm{M} \mathrm{H}_{2} \mathrm{SO}_{4}$ & $242(1.0 \mathrm{~A} / \mathrm{g})$ \\
\hline Pomelo peel & Carbonization & 2725 & $6 \mathrm{M} \mathrm{KOH}$ & $342(0.2 \mathrm{~A} / \mathrm{g})$ \\
\hline Poplar catkins & Carbonization/ $/ \mathrm{ZnCl}_{2}$ activation & 1463 & $1 \mathrm{M} \mathrm{H}_{2} \mathrm{SO}_{4}$ & $251(0.5 \mathrm{~A} / \mathrm{g})$ \\
\hline Chitosan & $\begin{array}{c}\text { Aerogel formation/ } \\
\text { carbonization/activation }\end{array}$ & 2435 & $6 \mathrm{M} \mathrm{KOH}$ & $197(0.2 \mathrm{~A} / \mathrm{g})$ \\
\hline Human hair & carbonization & 1306 & $6 \mathrm{M} \mathrm{KOH}$ & $340(1.0 \mathrm{~A} / \mathrm{g})$ \\
\hline $\begin{array}{l}\text { Chinese parasol } \\
\text { fluff }\end{array}$ & $\begin{array}{l}\text { Metal ion pre-treatment/ } \\
\text { two-step post-activation }\end{array}$ & 1449 & $2 \mathrm{M} \mathrm{H}_{2} \mathrm{SO}_{4}$ & $682(0.2 \mathrm{~A} / \mathrm{g})$ \\
\hline
\end{tabular}


Table S3 Comparison of the electrochemical properties of the as-made samples with other biomass carbon from recent references.

\begin{tabular}{|c|c|c|c|c|}
\hline Precursor & $C_{\mathrm{g}}$ & Measurements & Current density (A/g)/cycle & References \\
\hline & $(\mathbf{F} / \mathbf{g})$ & done at & number $/ C_{\mathrm{g}}$ retention $(\%)$ & \\
\hline Commercial cotton & 305 & $0.1 \mathrm{~A} / \mathrm{g}$ & $5 / 10000 / 98$ & 1 \\
\hline Bamboo char & 222 & $0.5 \mathrm{~A} / \mathrm{g}$ & ------------ & 2 \\
\hline Perilla frutescens & 270 & $0.5 \mathrm{~A} / \mathrm{g}$ & 2/10000/96.1 & 3 \\
\hline Sugar cane bagasse & 300 & $0.25 \mathrm{~A} / \mathrm{g}$ & $2 / 5000 / 90$ & 4 \\
\hline Salvia splendens & 294 & $1 \mathrm{~A} / \mathrm{g}$ & $2 / 20000 / 92.5$ & 5 \\
\hline Willow catkin & 285 & $1 \mathrm{~A} / \mathrm{g}$ & $5 / 10000 / 98$ & 6 \\
\hline Poplar catkins & 251 & $0.5 \mathrm{~A} / \mathrm{g}$ & 20/10000/99.5 & 7 \\
\hline Cattle bones & 258 & $5 \mathrm{~A} / \mathrm{g}$ & $10 / 5000 / 96.4$ & 8 \\
\hline Banana fibers & 74 & $0.5 \mathrm{~A} / \mathrm{g}$ & $0.5 / 500 / 89$ & 9 \\
\hline Pomelo peel & 342 & $0.2 \mathrm{~A} / \mathrm{g}$ & $10 / 1000 / 98$ & 10 \\
\hline Tufo & 262 & $0.5 \mathrm{~A} / \mathrm{g}$ & $3 / 10000 / 102$ & 11 \\
\hline Cotton & 175 & $1 \mathrm{~A} / \mathrm{g}$ & 20/5000/95 & 12 \\
\hline Lotus stems & 174 & $5 \mathrm{mV} / \mathrm{s}$ & $1 / 10000 / 72$ & 13 \\
\hline CCPF-750-15 & $\begin{array}{l}682 \\
397\end{array}$ & $0.2,1 \mathrm{~A} / \mathrm{g}$ & $10 / 10000 / 92.19$ & This work \\
\hline
\end{tabular}


Table S4 $R_{\mathrm{ct}}$ and $R_{\mathrm{S}}$ values of the as-prepared samples.

\begin{tabular}{lcc}
\hline Samples & $\boldsymbol{R}_{\mathrm{ct}}(\boldsymbol{\Omega})$ & $\boldsymbol{R}_{\mathrm{s}}(\boldsymbol{\Omega})$ \\
\hline CCPF-650-15 & 6.25 & 1.26 \\
CCPF-750-30 & 1.78 & 0.96 \\
CCPF-750-45 & 2.88 & 1.31 \\
CCPF-850-15 & & 1.37 \\
CCPF-950-15 & 4.06 & 1.21 \\
\hline
\end{tabular}


Table S5 The detailed information of the reported biochar sample.

\begin{tabular}{|c|c|c|c|c|c|}
\hline Precursor & $\begin{array}{l}\text { Treatment } \\
\text { conditions }\end{array}$ & $\begin{array}{c}\text { SSA } \\
\left(\mathrm{m}^{2} / \mathbf{g}\right)\end{array}$ & Electrolyte & $\begin{array}{l}\text { Energy } \\
\text { density } \\
(\mathrm{Wh} / \mathrm{kg})\end{array}$ & $\begin{array}{l}\text { Power } \\
\text { density } \\
\text { (W/kg) }\end{array}$ \\
\hline $\begin{array}{c}\text { Glucose@ rice } \\
\text { straw }\end{array}$ & $\begin{array}{l}\text { In situ decoration } \\
/ \mathrm{KOH} \text { activation }\end{array}$ & 1122 & $6 \mathrm{M} \mathrm{KOH}$ & 9.3 & 500 \\
\hline Pomelo peel & Carbonization & 2725 & $6 \mathrm{M} \mathrm{KOH}$ & 9.4 & 96 \\
\hline $\begin{array}{l}\text { Leonardite } \\
\text { fulvic } \\
\text { acid }\end{array}$ & $\begin{array}{c}\text { Solvent exchange/activation } \\
\text { process }\end{array}$ & 2807 & $6 \mathrm{M} \mathrm{KOH}$ & 13 & 60 \\
\hline $\begin{array}{l}\text { Sodium } \\
\text { alginate }\end{array}$ & $\begin{array}{c}\text { polymerization } \\
\text { carbonization/activation }\end{array}$ & 1695 & $\mathrm{H}_{2} \mathrm{SO}_{4}$-PVA gel & 3.8 & 246 \\
\hline $\begin{array}{c}\text { Bamboo } \\
\text { char }\end{array}$ & $\mathrm{K}_{2} \mathrm{FeO}_{4} /$ carbonization & 1732 & KOH-PVA gel & 6.68 & 100 \\
\hline $\begin{array}{l}\text { Prawn } \\
\text { shells }\end{array}$ & $\begin{array}{c}\text { Carbonization/KOH } \\
\text { activation }\end{array}$ & 1606 & $1 \mathrm{M} \mathrm{H}_{2} \mathrm{SO}_{4}$ & 6.5 & 1000 \\
\hline $\begin{array}{l}\text { Apricot } \\
\text { shell }\end{array}$ & $\begin{array}{l}\text { TIPS approach } \\
\text { KOH activation }\end{array}$ & 1790 & $1 \mathrm{M} \mathrm{H}_{2} \mathrm{SO}_{4}$ & 5.1 & 4150 \\
\hline $\begin{array}{l}\text { Peanut } \\
\text { shell }\end{array}$ & $\mathrm{ZnCl}_{2}$ activation & 1552 & $1 \mathrm{M} \mathrm{Et}_{4} \mathrm{NBF}_{4} / \mathrm{PC}$ & 19.3 & 1007 \\
\hline $\begin{array}{l}\text { Willow } \\
\text { catkins }\end{array}$ & $\begin{array}{l}\text { Carbonization } \\
\mathrm{KOH} \text { activation }\end{array}$ & 1776 & $1 \mathrm{M} \mathrm{LiPF}_{6}$ & 37.9 & 700 \\
\hline $\begin{array}{c}\text { Sodium } \\
\text { lignosulfonate }\end{array}$ & $\begin{array}{c}\text { Thermostabilization } \\
\text { carbonization }\end{array}$ & 1939 & $1 \mathrm{M} \mathrm{SBPBF}_{4} / \mathrm{PC}$ & 29.3 & 2000 \\
\hline Rice straw & $\begin{array}{l}\text { Carbonization } \\
\mathrm{KOH} \text { activation }\end{array}$ & 1007 & {$[\mathrm{EMIM}]\left[\mathrm{BF}_{4}\right]$} & 17.4 & 126 \\
\hline $\begin{array}{l}\text { Carboxymeth } \\
\text {-ylcellulose } \\
\text { sodium }\end{array}$ & $\mathrm{KNO}_{3}$ activation & 1773 & $1 \mathrm{M} \mathrm{TEASF}_{4} / \mathrm{AN}$ & 34.7 & 675 \\
\hline Birch wood & $\begin{array}{c}\text { Carbonization } \\
\mathrm{NaOH} \text { activation }\end{array}$ & 1456 & $\begin{array}{c}1 \mathrm{M} \\
\left(\mathrm{C}_{2} \mathrm{H}_{5}\right)_{4} \mathrm{NBF}_{4} / \mathrm{AN}\end{array}$ & 23.25 & 3600 \\
\hline Chinese parasol & Metal ion pre-treatment/ & 1449 & $2 \mathrm{M} \mathrm{H}_{2} \mathrm{SO}_{4}$ & 17.64 & 100 \\
\hline fluff & two-step post-activation & & $1 \mathrm{M} \mathrm{BMIMBF} 4 / \mathrm{AN}$ & 46.38 & 300 \\
\hline
\end{tabular}




\section{References}

1 Fan, M.; Song, L.; Li, X.; Fan, L. Assembly of Graphene Aerogels into the 3D Biomass-Derived Carbon Frameworks on Conductive Substrates for Flexible Supercapacitors. Carbon 2017, 111, 658-666.

2 Gong, Y.; Li, D.; Luo, C.; Fu, Q.; Pan, C. Highly Porous Graphitic Biomass Carbon as Advanced Electrode Materials for Supercapacitors. Green Chem. 2017, 19, 4132-4140.

3 Liu, B.; Liu, Y.; Chen, H.; Yang, M.; Li, H. Oxygen and Nitrogen Co-Doped Porous Carbon Nanosheets Derived from Perilla Frutescens for High Volumetric Performance Supercapacitors. J. Power Sources 2017, 341, 309-317.

4 Rufford, T.E.; Hulicova-Jurcakova, D.; Khosla, K.; Zhu, Z.; Lu, G.Q. Microstructure and Electrochemical Double-Layer Capacitance of Carbon Electrodes Prepared by Zinc Chloride Activation of Sugar Cane Bagasse. J. Power Sources 2010, 195, 912-918.

5 Liu, B.; Yang, M.; Chen, H.; Liu, Y.; Yang, D.; Li, H. Graphene-Like Porous Carbon Nanosheets Derived from Salvia Splendens for High-Rate Performance Supercapacitors. J. Power Sources 2018, 397, 1-10.

6 Li, Y.; Wang, G.; Wei, T.; Fan, Z.; Yan, P. Nitrogen and Sulfur Co-Doped Porous Carbon Nanosheets Derived from Willow Catkin for Supercapacitors. Nano Energy 2016, 19, 165-175.

7 Gao, S.; Li, X.; Li, L.; Wei, X. A Versatile Biomass Derived Carbon Material for Oxygen Reduction Reaction, Supercapacitors and Oil/Water Separation. Nano Energy 2017, 33, 334-342.

8 Niu, J.; Shao, R.; Liang, J.; Dou, M.; Li, Z.; Huang, Y.; Wang, F. Biomass-Derived Mesopore-Dominant Porous Carbons with Large Specific Surface Area and High Defect Density as High Performance Electrode Materials for Li-ion Batteries and Supercapacitors. Nano Energy 2017, 36, 322-330.

9 Subramanian, V.; Luo, C.; Stephan, A.M.; Nahm, K.S.; Thomas, S.; Wei, B. Supercapacitors from Activated Carbon Derived from Banana Fibers. J. Phys. Chem. C 2007, 111, 7527-7531.

10Liang, Q.; Ye, L.; Huang, Z.-H.; Xu, Q.; Bai, Y.; Kang, F.; Yang, Q.-H. A Honeycomb-Like Porous Carbon Derived from Pomelo Peel for Use in High-Performance Supercapacitors. Nanoscale 2014, 6, 13831-13837.

11 Ouyang, T.; Cheng, K.; Yang, F.; Zhou, L.; Zhu, K.; Ye, K.; Wang, G.; Cao, D. From Biomass with Irregular Structures to 1D Carbon Nanobelts: a Stripping and Cutting Strategy to Fabricate High Performance Supercapacitor Materials. J. Mater. Chem. A 2017, 5, 14551-14551.

12 Wang, H.; Yi, H.; Zhu, C.; Wang, X.; Fan, H.J. Functionalized Highly Porous Graphitic Carbon Fibers for High-Rate Supercapacitive Electrodes. Nano Energy, 2015, 13 658-669. 
13Zhang, Y.; Liu, S.; Zheng, X.; Wang, X.; Xu, Y.; Tang, H.; Kang, F.; Yang, Q.; Luo, J. Biomass Organs Control the Porosity of Their Pyrolyzed Carbon. Adv. Funct. Mater. 2017, 27, 1604687-1604694. 